\title{
Rarefactions and Large Time Behavior for Parabolic Equations and Monotone Schemes
}

Eduard Harabetian $\star \star$

Department of Mathematics, Stanford University, Stanford, CA 94305, USA

\begin{abstract}
We consider the large time behavior of monotone semigroups associated with degenerate parabolic equations and monotone difference schemes. For an appropriate class of initial data the solution is shown to converge to rarefaction waves at a determined asymptotic rate.
\end{abstract}

\section{Introduction}

Our main point of interest is the large time behavior of two solution operators, one continuous, the other discrete, when acting on a certain class of initial data.

The continuous example is the solution to the class of degenerate parabolic equations of the type

$$
u_{t}+f(u)_{x}=A(u)_{x x}
$$

where $u$ is scalar, $f$ is convex and $A^{\prime}(u) \geqq 0$. When $A(u)=|u|^{\gamma} \cdot u, \gamma>0$, we have the convective porous medium equation.

The discrete example is the class of monotone difference schemes for the scalar conservation law ((1.1) with $A \equiv 0)$. We write the scheme in the following way:

$$
u^{n+1}(x)=u^{n}(x)-\lambda \Delta_{d}\left(g\left(u^{n}\left(x-p_{0} d\right), \ldots, u^{n}\left(x+q_{0} d\right)\right),\right.
$$

where we chose $x \in \mathbf{R}$ rather than on a mesh

$$
\lambda=\frac{\Delta x}{\Delta t}, \quad\left(\Delta_{d} u\right)(x)=u(x)-u(x-d), \quad p_{0} \geqq 0, \quad q_{0}>0, \quad d>0,
$$

and several conditions on the numerical flux $g$ will be specified. The parameter $d$ is not necessarily small.

* Supported by NSF Postdoctoral Fellowship Grant No. DMS 85-11476

This research was supported in part by the Institute for Math and its Applications with funds provided by the National Science Foundation

$\star \star$ Current address: Department of Mathematics, The University of Michigan, Ann Arbor, Michigan 48109-1003, USA 
The scalar conservation law

$$
u_{t}+f(u)_{x}=0
$$

is invariant under the transformation

$$
x=\frac{\tilde{x}}{v}, \quad t=\frac{\tilde{t}}{v}, \quad v>0,
$$

and it has continuous, self-similar solutions of the form

$$
r(x, t)=R\left(\frac{x}{t}\right)= \begin{cases}u_{-} & \frac{x}{t}<a\left(u_{-}\right) \\ a^{-1}\left(\frac{x}{t}\right) & a\left(u_{-}\right) \leqq \frac{x}{t} \leqq a\left(u_{+}\right), \\ u_{+} & a\left(u_{+}\right)<\frac{x}{t}\end{cases}
$$

where $a(u)=f^{\prime}(u)$ and $u_{-}<u_{+}$are the values at $\mp \infty$; these solutions are called rarefactions. With respect to the variables $\tilde{x}, \tilde{t}$ defined in (1.4) Eq. (1.1) changes to

$$
u_{\tilde{t}}+f(u)_{\tilde{x}}=v A(u)_{\tilde{x} \tilde{x}},
$$

and its solutions are close to solutions of (1.3) when $v$ is small.

For the monotone schemes in (1.2) this scaling procedure, in effect, changes $d$ to $v d$, and the consistency with (1.3) is merely a consequence of the consistency of $g$ with $f$.

We will prove that for a fairly large class of initial data, the error between solutions in (1.1) and (1.2) and the appropriate rarefactions tends to zero in $L^{p}, 1<p \leqq \infty$. More specifically $u=R+K$ and $|K|_{L^{p}(d x)} \leqq$ $c(\ln t)^{(1 / 2)+(1 / 2 p)} t^{-(1 / 2)+(1 / 2 p)}, 1 \leqq p \leqq \infty$, and the rate of decay for $K$, without the $\ln t$ term, is the real rate for Burger's equation (when $f(u)=1 / 2 u^{2}$ and $A(u)=u$ ).

In the next section we will prove a proposition which states the result in the more general framework of monotone semigroups that satisfy a consistency condition.

In $L^{1}$, the example of Burger's equation shows that we stay at a positive distance from rarefactions.

The complementary situation, when $u_{-}>u_{+}$and (1.1), (1.2) admit travelling waves, was treated in Ref. [3] and [1]. It was shown there that these travelling wave solutions attract in $L^{1}$ a large class of initial data.

In [4], there are results about the $L^{\infty}$ behavior of the equation $u_{t}+f(u)_{x}=c u_{x x}$, $c>0$, without a rate.

\section{Monotone Semigroups}

For $u_{-}<u_{+}$we define $U \subset L^{\infty}(R)$ by

$$
U=\left\{u \in L^{\infty}, u_{-} \leqq u(x) \leqq u_{+}, \int_{x<0}\left|\sup _{z \leqq x} u(z)-u_{-}\right| d x<\infty,\right.
$$




$$
\left.\int_{x>0}\left|\inf u(z)-u_{+}\right| d x<\infty\right\} .
$$

As in [1] we consider a semigroup $T(t), t \in R_{+}$or $Z_{+}$, defined on $U$, and satisfying:

(1) $u \leqq v$ a.e. $\Rightarrow T(t) u \leqq T(t) v$ a.e. (monotone),

(2) $u-v \in L^{1} \Rightarrow T(t) u-v \in L^{1}$ (preserves $L^{1}$ ),

(3) $u-v \in L^{1} \Rightarrow \int_{-\infty}^{+\infty} T(t) u-T(t) v=\int_{-\infty}^{+\infty} u-v$ (conservative),

(4) $T(t) \tau_{h}=\tau_{h} T(t), \quad \tau_{h} u=u(x-h)$ (translation invariant).

A Lemma of Crandall and Tartar [9] shows that, given (2) and (3), the property (1) is equivalent to

$$
|T(t) u-T(t) v|_{L^{1}} \leqq|u-v|_{L^{1}}, \quad \text { if } \quad u-v \in L^{1}\left(L^{1}-\text { contractive }\right) .
$$

With this we form $T_{\theta}^{\alpha}=\delta_{1 / h} T(\alpha) \delta_{h}$, where $0<\alpha \leqq 1, \delta_{h} u=u(h x)$ and note that $T_{h}^{\alpha}$ is also an $L^{1}$-contraction. If $t \in \mathbf{Z}_{+}$, then $\alpha$ is by definition equal to 1 .

The next condition makes $T(t)$ consistent with a self-similar solution. Suppose there exists $\rho(x) \in U$ which is Lipschitz continuous, $\rho^{\prime} \geqq 0,\left|\rho^{\prime}\right|_{\ell^{\infty}}<\infty$, and such that

$$
\left|T_{h}^{\alpha} \rho-\delta_{1 /(1+a h)} \rho\right|_{L^{1}} \leqq C h^{2} .
$$

Then,

Proposition. $\left|T(t) u-\delta_{1 / t} \rho\right|_{L^{p}} \leqq C(\ln t)^{(1 / 2)+(1 / 2 p)} t^{-(1 / 2)+(1 / 2 p)}, t \geqq 1,1 \leqq p \leqq \infty$, $u \in U$.

Remark. The constants $C$ are not the same and they don't depend on $h$ or $t$ etc. Before proving the proposition, a few remarks about (6): We note that an equation which is invariant under (1.4) has a solution operator $T(t)$ which satisfies $\delta_{1 / h} T(\alpha) \delta_{h}=T(\alpha h)$, and therefore the left-hand side of (6) is identically zero if $T(t) \rho=\delta_{1 /(1+h)} \rho$. For Eq. (1.1), (6) represents the following local condition:

Let $v$ satisfy

$$
\begin{gathered}
v_{t}+f(v)_{x}=h A(v)_{x x}, \\
v(0, x)=R(x)=r(x, 1)(\text { see } 1.5)
\end{gathered}
$$

Then

$$
|v(\alpha h, x)-r(1+\alpha h, x)|_{L^{1}} \leqq C h^{2} .
$$

Here $\rho(x)=R(x)$

For (1.2), to be consistent, we take

$$
\rho(x)=R\left(\frac{x}{\lambda d}\right) .
$$

Condition (6) now amounts to the requirement that the local truncation error for consistent monotone schemes is of $O\left(h^{2}\right)$ in $L^{1}$, where $h$ is the mesh-size. Since 
rarefactions are Lipschitz continuous with bounded derivatives, we will be able to prove this in Sect. 4.

Proof of Proposition. First, let $p=1$ and define:

$$
u^{t}(x)=(T(t) u)(t x)=\delta_{t} T(t) u
$$

We then have the identity:

$$
\left|u^{t}-\rho\right|_{L^{1}}=\frac{1}{t}\left|T(t) u-\delta_{1 / t} \rho\right|
$$

and what we need to show is:

$$
\left|u^{t}-\rho\right|_{L^{1}} \leqq C \frac{\ln t}{t}
$$

It suffices to consider $t=n \in Z^{+}$, since, with $t=n+\alpha$, for some $0<\alpha<1$,

$$
\begin{aligned}
\left|T(n+\alpha) u-\delta_{1 /(n+\alpha)} \rho\right|_{L^{1}} & \leqq\left|T(n) u-\delta_{1 / n} \rho\right|_{L^{1}}+\left|T(\alpha) \delta_{1 / n} \rho-\delta_{1 /(n+\alpha)} \rho\right|_{L^{1}} \\
& =\left|T(n) u-\delta_{1 / n} \rho\right|_{L^{1}}+n\left|\delta_{n} T(\alpha) \delta_{1 / n} \rho-\delta_{n /(n+\alpha)} \rho\right|_{L^{1}} \\
& \leqq\left|T(n) u-\delta_{1 / n} \rho\right|_{L^{1}}+\frac{C}{n} .
\end{aligned}
$$

Next, dropping the $L^{1}$ subscript, and by (2.3),

$$
\begin{aligned}
\left|u^{n+1}-\rho\right| & =\left|\delta_{n+1} T(n+1) u-\rho\right| \\
& =\frac{n}{n+1}\left|\delta_{n} T(1) \delta_{1 / n} \delta_{n} T(n) u-\delta_{n /(n+1)} \rho\right| \\
& \leqq \frac{n}{n+1}\left|T_{1 / n}^{1} u^{n}-T_{1 / n}^{1} \rho\right|+\frac{n}{n+1}\left|T_{1 / n}^{1} \rho-\delta_{n /(n+1)} \rho\right| \\
& \leqq \frac{n}{n+1}\left|u^{n}-\rho\right|+C \frac{n}{n+1} \cdot \frac{1}{n^{2}} \text { by }(6) .
\end{aligned}
$$

Assume that $\left|u^{j}-\rho\right| \leqq C(\ln j) / j$ for $2 \leqq j \leqq n$ with $C$ independent of $u_{0}$, where $\left|u_{0}-\rho\right|_{L^{1}} \leqq M$. This is true for $n=2$,

$$
\begin{aligned}
\left|u^{n+1}-\rho\right| & \leqq C \frac{\ln n}{n+1}+C \frac{1}{n(n+1)} \\
& =C\left(\frac{\ln (n+1)}{n+1}+\frac{1}{n+1}\left(\frac{1}{n}-\ln \left(1+\frac{1}{n}\right)\right)\right) \leqq C \frac{\ln (n+1)}{n+1},
\end{aligned}
$$

and the induction step is complete.

To prove the case $p=\infty$, we first observe that we can restrict our attention to $u \in U, u$ increasing, since for any $u \in U$, our definition of $U$ allows for two functions $\varphi_{l}, \varphi_{u} \in U$, increasing, such that $\varphi_{l} \leqq u \leqq \varphi_{u}$. The monotonicity of $T(t)$ then yields

$$
|T(t) u-r|_{L^{\infty}} \leqq\left|T(t) \varphi_{l}-r\right|_{L^{\infty}}+\left|T(t) u-T(t) \varphi_{l}\right|_{L^{\infty}}
$$




$$
\begin{aligned}
& \leqq\left|T(t) \varphi_{l}-r\right|_{L^{\infty}}+\left|T(t) \varphi_{u}-T(t) \varphi_{l}\right|_{L^{\infty}}, \quad \text { by Condition (1) } \\
& \leqq 2\left|T(t) \varphi_{l}-r\right|_{L^{\infty}}+\left|T(t) \varphi_{u}-r\right|_{L^{\infty}} .
\end{aligned}
$$

To continue the proof for $p=\infty$, we fix $x_{1}$ and let $l=\rho\left(x_{1}\right)-u^{t}\left(x_{1}\right)$ and without loss of generality let $l \geqq 0$. We also let $M=\left|\rho^{\prime}\right|_{L^{\infty}}$ and $x_{0}=x_{1}-l / M$. Then,

$$
\rho(x) \geqq u^{t}\left(x_{1}\right)+M\left(x-x_{0}\right), \quad x_{0} \leqq x \leqq x_{1},
$$

since they are equal at $x=x_{1}$ and the derivative of the function on the right side of the equality is always bigger.

Since $u^{t}(x)$ is increasing $(T(t)$ preserves monotonicity),

$$
u^{t}\left(x_{1}\right)+M\left(x-x_{0}\right) \geqq u^{t}\left(x_{1}\right) \geqq u^{t}(x), \quad x_{0} \leqq x \leqq x_{1},
$$

and therefore,

$$
\left|u^{t}-\rho\right|_{L^{1}} \geqq \int_{x_{0}}^{x_{1}}\left(\rho-u^{t}\right) d x \geqq \int_{x_{0}}^{x_{1}} M\left(x-x_{0}\right) d x=\frac{1}{2} \frac{l^{2}}{M} .
$$

And now, since the $L^{\infty}$ norm is invariant under $\delta_{t}$,

$$
\left|T(t) u-\delta_{1 / t} \rho\right|_{L^{\infty}}=\left|u^{t}-\rho\right|_{L^{\infty}} \leqq \sqrt{2 M\left|u^{t}-\rho\right|_{L^{\infty}}} \leqq C\left(\frac{\ln t}{t}\right)^{1 / 2} .
$$

Finally, in $L^{p}$

$$
\begin{aligned}
\left|T(t) u-\delta_{1 / t} \rho\right|_{L^{\infty}} & \leqq\left|T(t) u-\delta_{1 / t} \rho\right|_{L^{\infty}}^{1-1 / p}\left|T(t) u-\delta_{1 / t} \rho\right|_{L^{1}}^{1 / p} \\
& \leqq C(\ln t)^{(1 / 2 p)+(1 / 2)} t^{-(1 / 2)+(1 / 2 p)} .
\end{aligned}
$$

\section{Quasilinear Parabolic Equations}

We consider (1.1) when $A(u)$ is smooth in $\left(u_{-}, u_{+}\right)$, and it is differentiable with $A^{\prime}$ nonnegative and Lipschitz continuous in $\left[u_{-}, u_{+}\right]$. We have thus included the porous medium equation when $u_{-}=0$. The results in Ref. [2], and the extensions in Ref. [1] show that there exists a unique solution operator satisfying (1)-(5) of Sect. 2.

Volpert and Hudjaev regularize the equation by adding artificial viscosity and they obtain estimates independent of the viscosity parameter. In the statement of their theorem they need more smoothness on $A(u)$. Osher and Ralston overcome this difficulty by modifying the initial data.

We let $\tilde{u}$ satisfy the following equation which incorporates both regularizations:

$$
\begin{aligned}
\tilde{u}_{t}+f(\tilde{u})_{x} & =(A(\tilde{u})+v \tilde{u})_{x x}, \\
\tilde{u}(0, x) \in U_{\varepsilon} & =\left\{\varphi \in U: u_{-}+\varepsilon \leqq \varphi \leqq u_{+}-\varepsilon\right\} .
\end{aligned}
$$

Standard results on parabolic equations yield smooth classical solutions to (3.1) [5].

Our claim is that it suffices to verify property (6), i.e. Eq. (2.1) for smooth solutions $\tilde{u}$ with a constant independent of $\varepsilon$ and $v$. To show this we use the 
estimates in Ref. [2] together with the arguments in Ref. [1] which yield

$$
\int_{-\infty}^{+\infty}|u(x, t)-\tilde{u}(x, t)| w_{\lambda}(x) d x \leqq e^{K_{\lambda} t} \int|u(x, 0)-\tilde{u}(x, 0)| w_{\lambda}(x) d x,
$$

where

and

$$
w_{\lambda}(x)=\exp \left(-\lambda\left(1+x^{2}\right)^{1 / 2}\right)
$$

$$
K_{\lambda}=\lambda \sup \left(\left|f^{\prime}(u)\right|+(1+\lambda) A^{\prime}(u)\right)
$$

Now take $\tilde{u}(0, x)=R_{\varepsilon}(x)=r_{\varepsilon}(1, x)$, the rarefaction which connects $u_{-}+\varepsilon$ to $u_{+}-\varepsilon$. Then

$$
\begin{aligned}
|u(\alpha h, x)-r(1+\alpha h, x)|_{L^{1}\left(w_{\alpha} d x\right)} \leqq & |u(\alpha h, x)-\tilde{u}(\alpha h, x)|_{L^{1}\left(w_{\lambda} d x\right)} \\
& +\left|\tilde{u}(\alpha h, x)-r_{\varepsilon}(1+\alpha h, x)\right|_{L^{1}\left(w_{\lambda} d x\right)} \\
& +\left|r_{\varepsilon}(1+\alpha h, x)-r(1+\alpha h, x)\right|_{L^{1}\left(w_{d} d x\right)} \\
\leqq & e^{K_{\lambda} \alpha h}\left|r(1, x)-r_{\varepsilon}(1, x)\right|_{L^{1}\left(w_{\lambda} d x\right)}+C h^{2} \\
& +\left|r_{\varepsilon}(1+\alpha h, x)-r(1+\alpha h, x)\right|_{L^{1}\left(w_{\lambda} d x\right)} .
\end{aligned}
$$

Now let $\varepsilon \rightarrow 0$ and then $\lambda \rightarrow 0$.

It remains, therefore, to show Eq. (2.1) when $A(u)$ is smooth and $A^{\prime}(u) \geqq a_{0}>0$ in $\left[u_{-}, u_{+}\right]$and that the constant $C$ doesn't depend on $a_{0}$. This last part will become evident from the proof.

We let $\phi=v-r$. Then $\phi$ is a Lipschitz continuous function which satisfies

$$
\begin{gathered}
\phi_{t}+\left(f(r+\varphi)-(f(r))_{x}=h A(v)_{x x},\right. \\
\phi(0, x)=0, \quad \text { see }(2.1)) .
\end{gathered}
$$

We multiply (3.2) by a regularized sign function of $\phi^{1}$, which is the derivative of a regularized absolute value function denoted by $L_{\varepsilon}$ and defined as follows:

$$
L_{\varepsilon}(z)=\left\{\begin{array}{ll}
|z|-\frac{\varepsilon}{2}, & |z|>\varepsilon \\
\frac{1}{2 \varepsilon} z^{2}, & |z|<\varepsilon
\end{array} .\right.
$$

Then the regularized sign function is given by

$$
L_{\varepsilon}^{\prime}(z)=\left\{\begin{array}{ll}
\operatorname{sgn} z, & |z|>\varepsilon \\
\frac{1}{\varepsilon}, & |z|<\varepsilon
\end{array},\right.
$$

\footnotetext{
1 We thank the referee for suggesting the use of a regularized sign function. This replaces the original less elegant argument
} 
and, from (3.2) we obtain:

$$
\int_{0}^{h} \int_{-\infty}^{+\infty} L_{\varepsilon}(\phi)_{t}+\int_{0}^{h} \int_{-\infty}^{+\infty} L_{\varepsilon}^{\prime}(\phi)[f(r+\phi)-f(r)]_{x}=h \int_{0}^{h} \int_{-\infty}^{+\infty} L_{\varepsilon}^{\prime}(\phi) A(\phi+r)_{x x} .
$$

The first term on the left, in (3.3), is $\int_{-\infty}^{+\infty} L_{\varepsilon}(\phi)(h)$ which tends to $|\phi|_{L^{1}(\alpha x)}(h)$ as $\varepsilon \rightarrow 0$ by Lebesgue's Dominated Convergence Theorem.

The second term on the left, in (3.3), after integrating by parts, is equal to:

$$
-\int_{0}^{h} \int_{-\infty}^{+\infty} L_{\varepsilon}^{\prime \prime}(\phi) \phi_{x}[f(r+\phi)-f(r)]=-\int_{0}^{h} \int_{-\infty}^{+\infty} \frac{1}{\varepsilon} \chi_{|\phi|<\varepsilon}[f(\phi+r)-f(r)] \phi_{x} .
$$

The integrand above tends to zero pointwise and is dominated by $\sup _{u \in\left[u_{-}, u_{+}\right]} f^{\prime}(u) \cdot\left|\phi_{x}\right|$. Therefore, by the Dominated Convergence Theorem the integral tends to zero as $\varepsilon \rightarrow 0$.

Finally, we consider the term on the right in (3.3). After integrating by parts and differentiating, we obtain:

$$
-h \int_{0}^{h} \int_{-\infty}^{+\infty} L_{\varepsilon}^{\prime \prime}(\phi) \phi_{x} A^{\prime}(r+\phi)\left(\phi_{x}+r_{x}\right) \leqq-h \int_{0}^{h} \int_{-\infty}^{+\infty} L_{\varepsilon}^{\prime \prime}(\phi) \phi_{x} A^{\prime}(r+\phi) r_{x},
$$

since $L_{\varepsilon}^{\prime \prime}(\phi) \phi_{x}^{2} A^{\prime}(r+\phi) \geqq 0$. (We note that this is the only place where we used $A^{\prime} \geqq 0$ and that we didn't need $A^{\prime}$ strictly positive.)

We now have

$$
\left.-h \int_{0}^{h} \int_{-\infty}^{+\infty} L_{\varepsilon}^{\prime}(\phi)_{x} A(r)_{x}-h \int_{0}^{h} \int_{-\infty}^{+\infty} L_{\varepsilon}^{\prime \prime}(\phi)\left(A^{\prime}(r+\phi)-A^{\prime}(r)\right)\right) \phi_{x} r_{x} .
$$

The second term tends to zero as $\varepsilon \rightarrow 0$ by virtue of the same Dominated Convergence Theorem, and the first term is estimated by

$$
h \int_{0}^{h}\left|A(r)_{x}\right|_{B V(d x)}(\tau) d \tau \leqq h^{2} \sup _{0 \leqq \tau \leqq h}\left|A(r)_{x}\right|_{B V(d x)},
$$

where the $B V(d x)$ norm is defined by

$$
|g|_{B V(d x)}=\sup _{h>0} \frac{1}{h} \int_{-\infty}^{+\infty}|g(x+h)-g(x)| d x .
$$

In conclusion, after letting $\varepsilon \rightarrow 0$, (3.3) yields $|\phi|_{L^{1}(d x)}(h) \leqq C h^{2}$, which is the desired estimate.

We close this section with the example mentioned in the introduction which uses Burger's equation:

$$
\begin{gathered}
u_{t}+\left(\frac{1}{2} u^{2}\right)_{x}=u_{x x}, \\
u(0, x)=H(x)= \begin{cases}0 & x<0 \\
1 & x>0\end{cases}
\end{gathered}
$$


Here one can solve explicitly, using the Cole-Hopf transformation, and obtain

$$
u(t, x)=\frac{\int_{0}^{\infty} e^{[(x / t)-1] y / 2} e^{-y^{2} / 4 t} d y}{\int_{-\infty}^{+\infty} e^{y x / 2 t-y_{+} / 2} e^{-y^{2} / 4 t} d y},
$$

where

$$
y_{+}=\left\{\begin{array}{ll}
y & y \geqq 0 \\
0 & y<0
\end{array} .\right.
$$

Here the rarefaction is given by

$$
r(x, t)= \begin{cases}0 & \frac{x}{t} \leqq 0 \\ \frac{x}{t} & 0 \leqq \frac{x}{t} \leqq 1 . \\ 1 & \frac{x}{t} \geqq 1\end{cases}
$$

To obtain the asymptotic expansion of $u(x, t)$ for $x \leqq 0$ we let $s=x / t$ and integrate by parts in the numerator to obtain

$$
\int_{0}^{\infty} e^{(s-1) y / 2} e^{-y^{2} / 4 z} d y=\frac{2}{s-1}\left\{-1+O\left(\frac{1}{t}\right)\right\},
$$

where $O(1 / t)$ is uniform in $s \leqq 0$.

In the denominator the dominant term is:

$$
\int_{-\infty}^{0} \exp \left(\frac{1}{2} \frac{x}{t} y-\frac{y^{2}}{4 t}\right) d y=2 \sqrt{t} e^{(x / 2 \sqrt{ } t)^{2}} \int_{-\infty}^{-x / 2 \sqrt{ } t} e^{-y^{2}} d y .
$$

Therefore, one obtains

$$
u(t, x)=\frac{1}{\sqrt{t} e^{(x / 2 \sqrt{ } t)^{2}} \int_{-\infty}^{-x / 2 \sqrt{ } t} e^{-y^{2}} d y}+K,
$$

where $|K|_{L^{p}\left(\mathbb{R}^{-}\right)} \leqq c t^{-1+1 / 2 p}, R^{-}=(-\infty, 0), 1 \leqq p \leqq \infty$. One can therefore verify that

$$
|u-r|_{L^{p}(\mathbb{R})}(t) \geqq|u-r|_{\left.L^{p^{-}} \mathbb{R}^{-}\right)}(t) \geqq c_{0} t^{(1-p) / 2 p}, \quad 1 \leqq p \leqq \infty, \quad C_{0}>0 .
$$

\section{Monotone Difference Schemes}

We consider (1.2) and impose the following conditions on the numerical flux $g=g\left(u_{-p_{0}}, \ldots, u_{+q_{0}}\right)$, a function of $p_{0}+q_{0}+1$ variables:

(a) $g(u, \ldots, u)=f(u)$.

(b) $g$ is Lipschitz continuous everywhere and $\partial g / \partial u_{i}$ are Lipschitz continuous in the domain $u_{-p_{0}} \leqq u_{-p_{0}+1} \leqq \cdots \leqq u_{q 0}$. 
(c) The function $u_{0}-\lambda\left(g\left(u_{-p_{0}}, \ldots, u_{q_{0}}\right)-g\left(u_{-\left(p_{0}+1\right)}, \ldots, u_{q_{0}-1}\right)\right)$ is nondecreasing in each of its arguments $u_{-p_{0}-1}, \ldots, u_{q_{0}}$.

For example, the Engquist-Osher (upwind) and Lax-Friedricks (dissipative) schemes, all satisfy these conditions which imply properties (1) through (5) of Sect. 2 for the solution operator. Unfortunately, Godunov's upwind scheme does not satisfy the second part of $(6)^{2}$.

For simplicity of notation take $\lambda d=1$ so in (2.2) $\rho(x)=R(x)$. Then, condition (6) is equivalent to

$$
\left|r(1+h, x)-r(1, x)-\Delta_{d} g\left(r\left(1, x-p_{0} h\right), \ldots, r\left(1, x+q_{0} h\right)\right)\right|_{L^{1}} \leqq C h^{2},
$$

with $r(t, x)=R(x / t)$ from $(1.5)$.

First, one easily verifies that

$$
\left|r(1+h, x)-r(1, x)-h r_{t}(1, x)\right|_{L^{1}} \leqq C h^{2},
$$

since the expression inside the $L^{1}$ norm is compactly supported, always bounded by $C h$ and bounded by $C h^{2}$ in the smooth regions which are outside some neighborhoods of $a\left(u_{-}\right), a\left(u_{+}\right)$of measure less than $C h$.

Next, by the mean value theorem

$$
\begin{aligned}
& \Delta_{d} g\left(R\left(x-p_{0} h\right), \ldots, R\left(x+q_{0} h\right)\right) \\
& \quad=h \sum_{i=-p_{0}}^{q_{0}} \int_{0}^{1} \frac{\partial g}{\partial u_{i}}(\ldots \theta R(x-i h)+(1-\theta) R(x-(i+1) h) \ldots) d \theta R^{\prime}\left(x-\left(i+\eta_{i}\right) h\right),
\end{aligned}
$$

for some $0 \leqq \eta_{i} \leqq 1$,

$$
\begin{aligned}
= & h \sum_{i} \frac{\partial g}{\partial u_{i}}(R(x), \ldots, R(x)) \cdot R^{\prime}(x) \\
& +h \sum_{i} \int_{0}^{1} \frac{\partial g}{\partial u_{i}}(\ldots \theta R(x-i h)+(1-\theta) R(x-(i+1) h) \ldots) \\
& -\frac{\partial g}{\partial u_{i}}(R(x), \ldots, R(x)) d \theta R^{\prime}\left(x-\left(i+\eta_{i}\right) h\right) \\
& +h \sum_{\frac{\partial g}{\partial u_{i}}}(R(x), \ldots, R(x))\left(R^{\prime}\left(x-\left(i+\eta_{i}\right) h\right)-R^{\prime}(x)\right)=h f(R)_{x}+K_{0} .
\end{aligned}
$$

Because of our assumptions on $g$ and since $\left|R^{\prime}\right|_{B V} \leqq C$, we get $\left|K_{0}\right|_{L^{1}} \leqq C h^{2}$ and the result follows.

In closing, we wish to mention that the result of the Proposition yields the following $L^{p}$ rate of convergence to rarefactions for monotone schemes:

$$
\left|u^{h}-R\right|_{L^{p}} \leqq C h^{(1 / 2)+(1 / 2 p)}(\ln 1 / h)^{(1 / 2)+(1 / 2 p)}, \quad 1 \leqq p \leqq \infty,
$$

where $h$ is the mesh size (which is related to the number of iterations in time), and $u^{h}(0, x)=\varphi(x / h), \varphi \in U$.

${ }^{2}$ We thank the referee for pointing this out 
The well-known results on convergence of monotone schemes [6-8] hold for general $L^{1} \cap B V \cap L^{\infty}$ initial data. Our rate of convergence, $h \ln (1 / h)$ in $L^{1}$, is an improvement over the previous rate, $h^{1 / 2}$, given in Refs. $[6,8]$. This is because, for our special case, it was possible to adopt a more direct type of proof of convergence.

\section{References}

1. Osher, S., Ralston, J.: $L^{i}$ Stability of travelling waves with applications to convective porous media flow. Commun. Pure. Appl. Math. 35, 737-751 (1982)

2. Volpert, A. I., Hudjaev ${ }_{2}$ S. I.: Cauchy's problem for degenerate second order quasilinear parabolic equations. Math. USSR Sb. 7, 365-387 (1969)

3. Jennings, G.: Discrete shocks. Commun. Pure. Appl. Math. 27, 25-37 (1974)

4. Il' in, A. M., Oleinik, O. A.: Behavior of the solutions of the Cauchy problem for certain quasilinear equations for unbounded increase of the time. Am. Math. Soc. Transl. Series 2, 42, 19-23 (1964)

5. Friedman, A.: Partial differential equations of parabolic type. New York: Prentice Hall 1964

6. Kuznetsov, N. N.: On Stable methods for solving non-linear first order partial differential equations in the class of discontinuous functions. Topics in numerical analysis, III (Proc. Roy. British Acad. Conf., Trinity Coll., Dublin 1976), pp. 183-197

7. Crandall, M., Majda, A.: Monotone difference approximations for scalar conservation laws. Math. Comp. 34, 1-22 (1980)

8. Sanders, R.: On convergence of monotone finite difference schemes with variable spatial differencing. Math. Comp. 40, 91-106 (1983)

9. Crandall, M., Tartar, L.: Some relations between nonexpansive and order preserving mappings. Proc. Am. Math. Soc. 78, 385-390 (1980)

Communicated by A. Jaffe

Received April 10, 1987; in revised form August 3, 1987 